\title{
Holography versus Correspondence principle: eternal Schwarzschild-anti-de Sitter geometry
}

\author{
Slava Emelyanov* \\ Arnold Sommerfeld Center for Theoretical Physics, \\ Ludwig Maximilian University (LMU), \\ 80333 Munich, Germany
}

(Dated: August 9, 2021)

\begin{abstract}
It is shown that the correspondence principle and the holographic principle are incompatible in the background of an eternal Schwarzschild-anti-de Sitter geometry. The argument is based on the observation that algebraic structures of local quantum field and CFT operators are not equivalent. This implies, in particular, the bulk CFT must be singular near the black-hole horizon. A CFT Hilbert space representation is elaborated which may correspond to the AdS black hole in the dual theory.
\end{abstract}

Keywords: black holes, correspondence principle, holography, AdS/CFT

*Electronic address: viacheslav.emelyanov@physik.uni-muenchen.de 


\section{INTRODUCTION}

The holographic principle states that the physical quantum gravity degrees of freedom are located on a co-dimension one hypersurface of a given spacetime [1, 2]. The AdS/CFT correspondence [3 5] is regarded in particular as a concrete realization of this principle. Specifically, the quantum gravity degrees of freedom are identified with rays in the conformal field theory (CFT) Hilbert space defined on the boundary $\partial \mathcal{M}$ of anti-de Sitter space (AdS). AdS spacetime is denoted as $\mathcal{M}_{1}$ below. The duality is further generalised to all geometries being asymptotically AdS space, among of which is the Schwarzschild-AdS black hole denoted as $\mathcal{M}_{2}$ in the following.

Having a boundary CFT field operator algebra, $\mathcal{O}_{\partial \mathcal{M}}$, one can construct its bulk analog $\mathcal{O}_{\mathcal{M}}$ [6 8]. The bulk CFT algebra $\mathcal{O}_{\mathcal{M}_{2}}$ is thus treated as a set of all possible observables (if self-adjoint and gauge invariant) in the Schwarzschild-AdS geometry. One may expect that quantum gravity should reduce to sufficiently well-established local quantum field theory (LQFT) defined on a fixed geometrical background. This is a semi-classical limit of quantum gravity, where gravity is governed by a classical theory, i.e. general relativity, and matter fields and perturbations of the metric are quantised. This reduction of quantum gravity to quantum field theory in curved (classical) spacetimes is a particular type of the correspondence principle. ${ }^{1}$

Local quantum field theory in globally hyperbolic spacetime is described by an algebra $\mathcal{A}_{\mathcal{M}}$ represented in a certain Hilbert space [12 14]. According to the correspondence principle, one should expect there exists a certain CFT algebra $\mathcal{O}_{\mathcal{M}}$ which is isomorphic to $\mathcal{A}_{\mathcal{M}}$ in the semi-classical limit. Specifically, this implies

$$
\left[\hat{O}_{\mathcal{M}}(x), \hat{O}_{\mathcal{M}}\left(x^{\prime}\right)\right]=\left[\hat{A}_{\mathcal{M}}(x), \hat{A}_{\mathcal{M}}\left(x^{\prime}\right)\right]
$$

for any $\hat{O}_{\mathcal{M}}(x) \in \mathcal{O}_{\mathcal{M}}$ and $\hat{A}_{\mathcal{M}}(x) \in \mathcal{A}_{\mathcal{M}}$, where $x, x^{\prime} \in \mathcal{M} \cup \partial \mathcal{M}$. Moreover, a unitary equivalence of theories should be then expected as well. In other words, the CFT Hilbert space representation of $\mathcal{O}_{\mathcal{M}}$ must be unitarily equivalent to that of $\mathcal{A}_{\mathcal{M}}$. The isomorphism between these theories is well-established in anti-de Sitter space [15-17].

A physical Hilbert space representation of field operators corresponds to a choice of a physical vacuum. In the AdS black-hole geometry, the Hartle-Hawking state, $\left|\Omega_{2}\right\rangle$, is supposed to be a physical one as being non-singular on the black-hole horizons. The regularity of $\left|\Omega_{2}\right\rangle$ allows thus to have a self-consistent treatment of quantum gravity in the semi-classical regime. A CFT state, $\left|\partial \Omega_{2}\right\rangle$, is an "analog" of the Hartle-Hawking state, but for $\mathcal{O}_{\mathcal{M}_{2}}$. It is expected to be a pure high-energy state which is thermal at the semi-classical approxima-

\footnotetext{
${ }^{1}$ Rigorously speaking, it is not a correspondence between quantum and classical theories, but this is a correspondence between a strongly fluctuating regime of quantum gravity and its weak-field/low-energy limit, when one is allowed to treat geometry as approximately classical, i.e. spacetime is classical up to its perturbations which are still quantised [9 11$]$.
} 
tion [7]. Roughly speaking, if the boundary CFT theory is in $\left|\partial \Omega_{2}\right\rangle$, then the bulk geometry should correspond to $\mathcal{M}_{2}$ according to AdS/CFT.

A question is posed in this paper whether the correspondence principle and holography are compatible with each other in the eternal Schwarzschild-AdS geometry. We will show below that there is no algebraic isomorphism between the operator algebras $\mathcal{A}_{\mathcal{M}_{2}}$ and $\mathcal{O}_{\mathcal{M}_{2}}$, i.e. $\mathcal{A}_{\mathcal{M}_{2}} \neq \mathcal{O}_{\mathcal{M}_{2}}$. We will also elaborate on properties of the Hilbert space constructed on the state $\left|\partial \Omega_{2}\right\rangle$.

The outline of this paper is as follows. In Sec. III, two theories based on $\mathcal{A}_{\mathcal{M}_{2}}$ and $\mathcal{O}_{\mathcal{M}_{2}}$ are separately considered. In Sec. IIA, we will briefly review local quantum field theory in the Schwarzschild-AdS geometry. In Sec. IIB, we will revisit a description of the AdS black hole by two CFT operator algebras represented on a factorised product of two CFT Hilbert spaces. The main point will be that although $\left|\partial \Omega_{2}\right\rangle$ can be represented as the thermal-field double state, this does not imply $\left|\partial \Omega_{2}\right\rangle$ belongs to a factorised product of the Hilbert spaces in quantum field theory. In Sec. IC, we will argue that the description should be given by a single CFT algebra represented on the state $\left|\partial \Omega_{2}\right\rangle$.

In Sec. III, these theories are treated together. In Sec. IIIA, it is pointed out that the Tomita-Takesaki theorem broadly employed to describe a Schwarzschild-AdS black-hole interior by CFT implies an extra geometrical construction on the black-hole boundary. In Sec. IIIB, it is shown that the operator algebras $\mathcal{A}_{\mathcal{M}_{2}}$ and $\mathcal{O}_{\mathcal{M}_{2}}$ are not algebraically isomorphic. This leads to an inconsistency when one simultaneously requires both the correspondence principle and the holographic principle in the semi-classical regime.

In Sec. IV, we will summarise our results and conclude.

Throughout this paper the fundamental constants are set to unity, $c=G=k_{\mathrm{B}}=\hbar=1$.

\section{ALGEBRAS $\mathcal{A}_{\mathcal{M}_{2}}$ AND $\mathcal{O}_{\mathcal{M}_{2}}$ AND THEIR REPRESENTATIONS}

According to (extrapolate) AdS/CFT, quantum field theory in the Schwarzschild-AdS geometry can be constructed either through a quantisation of a classical field defined inside it or through pulling the CFT operators from its boundary. In other words, one has at a disposal at least two operator algebras, i.e $\mathcal{A}_{\mathcal{M}_{2}}$ and $\mathcal{O}_{\mathcal{M}_{2}}$, respectively. One may expect these two theories are equivalent at the semi-classical approximation. In this section, we will separately discuss these two theories outside of the AdS black hole.

\section{A. Single $*$-algebra and its representations}

A quantum field $\hat{\Phi}(x)$, where $x \in \mathcal{M}$, is an operator-valued distribution. To have welldefined operators built out of it on a certain Hilbert space, it has to be averaged or smeared out over a set of test functions $\{f(x)\}$, i.e. functions being smooth and of a compact support. The field operator algebra $\mathcal{A}_{\mathcal{M}}$ is a set composed of an identity operator $\hat{\mathbf{1}}$ and operators 
generated by $\hat{\Phi}(f)$ with its non-linear combinations. It has a structure of a unital *-algebra, where the $*$-operation is an involution mapping an element of $\mathcal{A}_{\mathcal{M}}$ into another its element. ${ }^{2}$ Quantum states are defined as linear, positive and normalized functionals on $\mathcal{A}_{\mathcal{M}}$. A basic reference is [18] and recent reviews [12 14] devoted to local quantum field theory in curved spacetimes.

To fix definitions and notations used below, we will briefly review certain aspects of a (linear) quantum scalar field in the background of the eternal Schwarzschild-AdS geometry.

The eternal Schwarzschild-AdS black hole is obtained through the Kruskal extension of the Schwarzschild black hole in AdS space. We will denote this geometry as $\mathcal{K}$. A part of it corresponds to $\mathcal{M}_{2}^{\prime} \cup \mathcal{M}_{2}$, where $\mathcal{M}_{2}^{\prime}$ is a causal complement of $\mathcal{M}_{2}$. In other words, it is the "left" outside region of the black hole, whereas $\mathcal{M}_{2}$ is the "right" outside region of the hole (see fig. 1). One may then choose a subset of the test functions with vanishing support in either $\mathcal{M}_{2}$ or $\mathcal{M}_{2}^{\prime}$. This leads to a splitting of the operator algebra $\mathcal{A}_{\mathcal{K}}$ into a product of two factor subalgebras, i.e. $\mathcal{A}_{\mathcal{M}_{2}^{\prime}} \otimes \mathcal{A}_{\mathcal{M}_{2}}$. This procedure is analogous to that in the eternal Schwarzschild-Minkowski geometry [19 21] and [22].

A physical Hilbert space representation, $\mathcal{H}$, of the algebra $\mathcal{A}_{\mathcal{K}}$ is constructed on the Hartle-Hawking state $\left|\Omega_{2}\right\rangle$. This representation is in particular characterised by splitting the field operator $\hat{\Phi}(x)$ into a sum of two operators, i.e.

$$
\hat{\Phi}(x)=\hat{a}(x)+\hat{a}^{\dagger}(x),
$$

where $\hat{a}(h)$ and $\hat{a}^{\dagger}(h)$ are interpreted as the Hartle-Hawking particle annihilation and creation operators corresponding to a wave packet $h(x)$ being a solution of a scalar field equation, where

$$
\hat{a}(h) \equiv i \int_{\Sigma} d \Sigma_{\mu} \sqrt{-g(x)} g^{\mu \nu}(x)\left(h^{*}(x) \nabla_{\nu} \hat{\Phi}(x)-\hat{\Phi}(x) \nabla_{\nu} h^{*}(x)\right),
$$

where $\Sigma$ is a Cauchy surface, and star denotes a complex conjugation. The wave packet $h(x)$ when restricted to the black-hole horizons is positive frequency with respect to $\partial_{U}$ and $\partial_{V}$, where $U$ and $V$ are the Kruskal coordinates in which the horizons are at $U=0$ and $V=0$.

On the other hand, one may choose another wave packet $h(x)$ to be positive frequency one with respect to $\partial_{u}$ and $\partial_{v}$, where $u$ and $v$ are the retarded and advanced Schwarzschild coordinates. One can thus also write

$$
\hat{\Phi}(x)=\hat{b}_{\mathrm{L}}(x)+\hat{b}_{\mathrm{R}}(x)+\text { H.c. },
$$

where the operator $\hat{b}_{\mathrm{R}}(x)$ and its Hermitian conjugate have zero support in $\mathcal{M}_{2}^{\prime}$, while $\hat{b}_{\mathrm{L}}(x)$ and its Hermitian conjugate in $\mathcal{M}_{2}$. In other words, one rewrites $\mathcal{A}_{\mathcal{K}}$ as a product of two commuting sets of field operators $\mathcal{A}_{\mathcal{M}_{2}^{\prime}}$ and $\mathcal{A}_{\mathcal{M}_{2}}$ generated by "left" and "right"

\footnotetext{
${ }^{2}$ If one specifies a particular Hilbert space representation of the algebra, the involution is then identified with the Hermitian conjugation.
} 

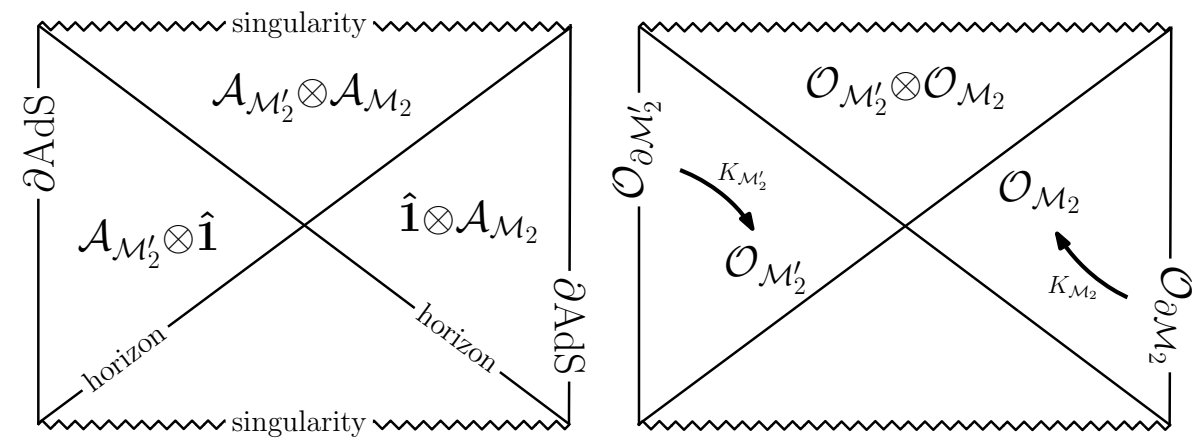

FIG. 1: A sketch of the Kruskal extension of the Schwarzschild-AdS black hole.

operators, respectively. One may further define two states $\left|\Omega_{\mathrm{L}}\right\rangle$ and $\left|\Omega_{\mathrm{R}}\right\rangle$ generating $\mathcal{H}_{\mathrm{L}}$ and $\mathcal{H}_{\mathrm{R}}$ and being vacua for $\hat{b}_{\mathrm{L}}(x)$ and $\hat{b}_{\mathrm{R}}(x)$, respectively. These states are the "left" and "right" Boulware vacua. Thus, we introduce a Hilbert space representation of the algebra on a factorized product, i.e. $\mathcal{H}_{\mathrm{L}} \otimes \mathcal{H}_{\mathrm{R}}$.

The Hilbert spaces $\mathcal{H}$ and $\mathcal{H}_{\mathrm{L}} \otimes \mathcal{H}_{\mathrm{R}}$ cannot be unitarily equivalent in LQFT:

$$
\mathcal{H} \nRightarrow \mathcal{H}_{\mathrm{L}} \otimes \mathcal{H}_{\mathrm{R}} \quad \text { or } \quad \mathcal{H} \perp \mathcal{H}_{\mathrm{L}} \otimes \mathcal{H}_{\mathrm{R}}
$$

This can be explained in terms of the type III property of factor subalgebras $\mathcal{A}_{\mathcal{M}_{2}^{\prime}}$ and $\mathcal{A}_{\mathcal{M}_{2}}$ of $\mathcal{A}_{\mathcal{K}}$ (see, for instance, [23]). It essentially means that the representation $\mathcal{H}$ of $\mathcal{A}_{\mathcal{K}}$ does not factorize into the product $\mathcal{H}_{\mathrm{L}} \otimes \mathcal{H}_{\mathrm{R}}$ under the factorization of $\mathcal{A}_{\mathcal{K}}$ into the factor subalgebras $\mathcal{A}_{\mathcal{M}_{2}^{\prime}}$ and $\mathcal{A}_{\mathcal{M}_{2}}$. This property has been recently emphasized in [11] in applications to the entanglement in LQFT.

The type III property of those factor subalgebras can be demonstrated as follows. Slightly generalising [19] to the asymptotically AdS case, one may rewrite the Hartle-Hawking state as a thermal-field double state [24], i.e.

$$
\left|\Omega_{2}\right\rangle=\frac{1}{Z^{\frac{1}{2}}} \prod_{\omega l m} \sum_{n=0}^{+\infty} e^{-\beta E_{\omega, n} / 2}\left|n_{\mathrm{L}}\right\rangle \otimes\left|n_{\mathrm{R}}\right\rangle, \quad \text { where } \quad E_{\omega, n} \equiv \omega n
$$

and $Z$ is a normalization factor, such that $\left\langle\Omega_{2} \mid \Omega_{2}\right\rangle=1$. The frequency $\omega>0$ is defined with respect to the Killing vector $K_{2}$, and $l, m$ are the orbital and magnetic numbers referring to a particular representation of the rotational symmetry of the black hole. The inverse temperature $\beta$ is given by $1 / T_{\mathrm{HP}}$, where $T_{\mathrm{HP}}$ is the Hawking-Page (HP) temperature [25]. The states entering the right-hand side of (6) are defined as

$$
\left|n_{\mathrm{L}}\right\rangle \equiv \frac{1}{\sqrt{n !}}\left(\hat{b}_{\mathrm{L}, \omega l m}^{\dagger}\right)^{n}\left|\Omega_{\mathrm{L}}\right\rangle
$$

and the same for $\left|n_{\mathrm{R}}\right\rangle$ with $\mathrm{L} \rightarrow \mathrm{R}$ in the above formula. It is worth noting that $n_{\mathrm{L}, \mathrm{R}}$ depend on $\omega, l$ and $m$. The state on the left-hand side of (6) is the only non-singular state on the black-hole horizons. The normalisation factor $Z$ is infinite (see, for instance, [26]). Thus, $\left|\Omega_{2}\right\rangle \notin \mathcal{H}_{\mathrm{L}} \otimes \mathcal{H}_{\mathrm{R}}$. Hence, the equation (66) is merely formal. This confirms (5). 
To summarize, any operator in $\mathcal{K}$ can be constructed by employing elements of the algebra $\mathcal{A}_{\mathcal{K}} \equiv \mathcal{A}_{\mathcal{M}_{2}^{\prime}} \otimes \mathcal{A}_{\mathcal{M}_{2}}$. There are two "natural" representations of $\mathcal{A}_{\mathcal{K}}$, namely $\mathcal{H}$ and $\mathcal{H}_{\mathrm{L}} \otimes \mathcal{H}_{\mathrm{R}}$. The former is built on the Hartle-Hawking vacuum $\left|\Omega_{2}\right\rangle$, whereas the latter on the Boulware vacua, i.e. $\left|\Omega_{\mathrm{L}}\right\rangle \otimes\left|\Omega_{\mathrm{R}}\right\rangle$. These are unitarily inequivalent (there is no unitary operator mapping these into each other), i.e. $\mathcal{H} \neq \mathcal{H}_{\mathrm{L}} \otimes \mathcal{H}_{\mathrm{R}}$.

\section{B. Two copies of CFT and their representations}

Following the proposal of $[8,27]$, physics in the eternal Schwarzschild-AdS geometry is described by two non-interacting (independent) CFT algebras represented on a factorised product of two CFT Hilbert spaces on each AdS boundary. Thus, the physical degrees of freedom are supported on two causally separated boundaries. These degrees of freedom are represented by rays in CFT Hilbert spaces. These will be denoted as $\partial \mathcal{H}_{\mathrm{L}}$ and $\partial \mathcal{H}_{\mathrm{R}}$. Two CFT states corresponding to these Hilbert spaces will be denoted as $\left|\partial \Omega_{\mathrm{L}}\right\rangle$ and $\left|\partial \Omega_{\mathrm{R}}\right\rangle$, respectively. At the semi-classical approximation, one actually deals with a thermal gas of excitations belonging to the Hilbert space $\partial \mathcal{H}_{\mathrm{L}} \otimes \partial \mathcal{H}_{\mathrm{R}}$ at the Hawking-Page temperature (see, for instance, [28]).

One may expect that the bulk CFT algebra represented in $\partial \mathcal{H}_{\mathrm{L}} \otimes \partial \mathcal{H}_{\mathrm{R}}$ reduces to local quantum field theory in the large $N$ limit, where $N$ refers to a degree of the gauge group characterising conformal field theory on the AdS boundary. This is a sort of the Bohr principle stating that a more fundamental theory (quantum gravity) contains a less fundamental one (semi-classical quantum field theory) in a certain limit $(N \rightarrow \infty)$. According to AdS/CFT extended to the eternal AdS black hole, one has $\mathcal{H}_{\mathrm{L}, \mathrm{R}} \cong \partial \mathcal{H}_{\mathrm{L}, \mathrm{R}}$. Since $\mathcal{H} \neq \mathcal{H}_{\mathrm{L}} \otimes \mathcal{H}_{\mathrm{R}}$, the bulk CFT algebra $\mathcal{O}_{\mathcal{M}_{2}}$ represented on $\partial \mathcal{H}_{\mathrm{R}}$ is not unitarily equivalent to the local algebra $\mathcal{A}_{\mathcal{M}_{2}}$ representation on the Hartle-Hawking state.

An entangled state considered in [8, 27] and leading to the Hilbert space $\partial \mathcal{H}$ is presumably identified with the Hartle-Hawking vacuum, such that $\mathcal{H} \cong \partial \mathcal{H}$. At least, it seems to be a motivation for selecting this state. In other words, one implicitly assumes by that the representations of the field operators $\mathcal{A}_{\mathcal{M}_{2}}$ and $\mathcal{O}_{\mathcal{M}_{2}}$ are unitarily equivalent in the semiclassical limit. $^{3}$

To summarize, the representation of $\mathcal{O}_{\mathcal{K}} \equiv \mathcal{O}_{\mathcal{M}_{2}^{\prime}} \otimes \mathcal{O}_{\mathcal{M}_{2}}$ in $\mathcal{K}$ must be $\partial \mathcal{H}$ which cannot be understood as a tensor product $\partial \mathcal{H}_{\mathrm{L}} \otimes \partial \mathcal{H}_{\mathrm{R}}$ of two (ordinary) CFT Hilbert spaces. In other words, these are unitarily inequivalent. The eternal-black-hole nucleation is a non-unitary process $\partial \mathcal{H}_{\mathrm{L}} \otimes \partial \mathcal{H}_{\mathrm{R}} \rightarrow \partial \mathcal{H}$. Usually, this kind of dynamics corresponds to phase transitions. This fits well to the idea of having the Hawking-Page phase transition.

\footnotetext{
${ }^{3}$ Note that the algebraic isomorphism between operator algebras $\mathcal{A}_{\mathcal{M}_{2}}$ and $\mathcal{O}_{\mathcal{M}_{2}}$ is here assumed. This issue will be investigated in detail below.
} 


\section{Single CFT and its representations}

Recently there was pronounced a proposal to describe the eternal Schwarzschild-AdS geometry by a single CFT [29]. Its possible realisation for different types of AdS black holes is demonstrated below in this section. We will show that a description by a single CFT is always implicitly present, although one introduces two copies of CFT. This observation follows from a state chosen for the CFT operators [8, 27].

BTZ black hole. To gain insight of how that can be done, let us consider the topological black hole in three-dimensional AdS space, i.e. the Bañados-Teitelboim-Zanelli (BTZ) black hole [30] (see [31] for a review). ${ }^{4}$

The BTZ black hole is actually described by a single CFT algebra defined on the boundary of $\mathrm{AdS}_{3}$. To see this one needs first to imagine that the CFT algebra in $\mathrm{AdS}_{3}$ space is split in two factor subalgebras with supports on the "left" and "right" AdS-Rindler patches of the $\mathrm{AdS}_{3}$ boundary, respectively. A CFT state to be non-singular on the Rindler horizons is the ordinary CFT vacuum (see fig. 2). One then takes the spatial Rindler coordinate be periodic. By doing this, one constructs the BTZ black hole. Thus, the physical Hilbert space representation of the single CFT algebra is constructed on the ordinary CFT vacuum (up to a quotient $[32])^{5}$ and generating the Hilbert space representation $\partial \mathcal{H}$.

Hence, two CFT algebras defined on each boundary of the BTZ black hole are factors of the single CFT algebra defined on the $\mathrm{AdS}_{3}$ boundary (up to the quotient). It is analogous to the splitting of $\mathcal{A}_{\mathcal{K}}$ into $\mathcal{A}_{\mathcal{M}_{2}^{\prime}} \otimes \mathcal{A}_{\mathcal{M}_{2}}$ discussed in Sec. II

A problem of no holographic description of the BTZ black hole pointed out in [34] appears only if one chooses an improper CFT algebra representation, i.e. $\partial \mathcal{H}_{\mathrm{L}} \otimes \partial \mathcal{H}_{\mathrm{R}}$, This representation of the field operators implies the absence of non-local vacuum correlations between casually unrelated regions (e.g., no Reeh-Schlieder property). As discussed above, the quantum gravity degrees of freedom must be associated with rays in $\partial \mathcal{H}$, rather than in that factorised product.

Schwarzschild-AdS black hole. The AdS black-hole nucleation is a quantum gravity process of the Hawking-Page phase transition from AdS space filled with a thermal gas to the eternal AdS black-hole geometry [25]. Let us imagine a flat space composed of two boundaries of the AdS black hole, i.e. $\partial \mathcal{K} \equiv \partial \mathcal{M}_{2}^{\prime} \cup \partial \mathcal{M}_{2}$, each of which is three-dimensional compactified Minkowski spacetime. The Schwarzschild time translation operator $K_{2}=\partial_{t_{\mathrm{S}}}$

\footnotetext{
${ }^{4}$ There is apparently a misuse of terminology in the literature in referring to the BTZ black hole as the eternal Schwarzschild-AdS one. To avoid any possible confusions, these two types of the black holes are distinguished throughout the paper.

${ }^{5}$ A certain quotient of AdS hyperboloid corresponding to an evolving universe was also discussed in [32]. A quantum field theory in this background has been studied in [33].
} 

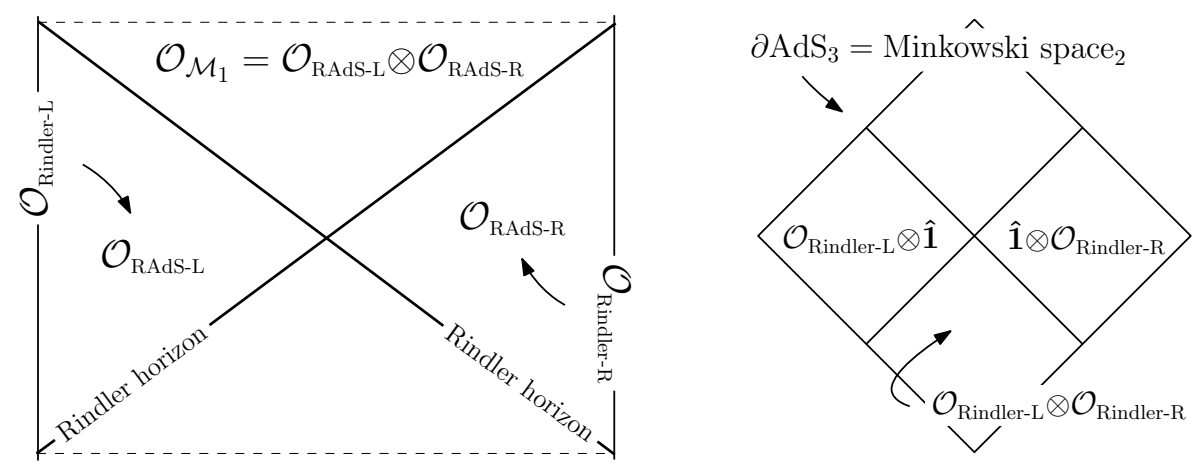

FIG. 2: "Left" and "right" AdS-Rindler (RAdS) patch in $\mathrm{AdS}_{3}$ and their boundary $\partial \mathrm{AdS}_{3}$. The CFT algebra $\mathcal{O}_{\partial A d S}$ can be represented as a product of two factor subalgebras, i.e. $\mathcal{O}_{\text {Rindler-L }} \otimes \mathcal{O}_{\text {Rindler-R. }}$. The CFT or Minkowski vacuum probed by either $\mathcal{O}_{\text {Rindler-L }}$ or $\mathcal{O}_{\text {Rindler-R }}$ responses as a thermal state, i.e. the vacuum two-point function for each of the factor subalgebras satisfies the Kubo-Martin-Schwinger condition with respect to the boost generators of the conformal group.

can be employed to set frequency modes in $\partial \mathcal{M}_{2}^{\prime} \cup \partial \mathcal{M}_{2}$. However, there are two possible unitarily inequivalent choices of the Hilbert space representation: either one considers a representation $\partial \mathcal{H}$ built on $\left|\partial \Omega_{2}\right\rangle$ or a representation $\partial \mathcal{H}_{\mathrm{L}} \otimes \partial \mathcal{H}_{\mathrm{R}}$ defined with respect to $\left|\partial \Omega_{\mathrm{L}}\right\rangle \otimes\left|\partial \Omega_{\mathrm{R}}\right\rangle$.

Each of $\partial \mathcal{M}_{2}^{\prime}$ and $\partial \mathcal{M}_{2}$ should be the whole three-dimensional Minkowski manifold. This can be shown at least in two ways. First, the boundary of AdS space, $\partial \mathcal{M}_{1}(\equiv \partial \mathcal{M})$, is compactified Minkowski space. The boundary of the Schwarzschild-AdS black hole, $\partial \mathcal{M}_{2}$, must fit $\partial \mathcal{M}$ to have the Hawking-Page transition. Second, the conformal Killing algebra of $\mathrm{AdS}_{4}$ space is given by $\mathfrak{s o}(2,4)$. Its generators are $L_{\mu \nu}, L_{\mu 4}$ and $L_{\mu 5}$, where $\mu, \nu \in\{0,1,2,3\}$. Among of these vectors, $L_{\mu 5}=\left\{L_{05}, L_{i 5}\right\}$ and $L_{\mu \nu}=\left\{L_{i j}, L_{0 i}\right\}$ are generators of the AdS isometry algebra, where $i, j$ run from 1 to 3. The Schwarzschild-AdS geometry has the fourdimensional Killing algebra, such that its Killing vector $K_{2}$ commutes with the generators of the rotations. The vectors $L_{05}$ and $L_{i j}$ are the only commuting generators of $\mathfrak{s o}(2,3)$. Thus, the Schwarzschild time translation generator $K_{2}$ must be identified with the Killing vector $L_{05}$ of the AdS hyperboloid. ${ }^{6}$ That is $K_{2}=K_{1}$, where $K_{1}$ is the global AdS time translation operator. This was pointed out in [7]. Hence, $\partial \mathcal{M}_{2}$ is Minkowski space, i.e. $\partial \mathcal{M}$, in the so-called closed coordinates. In these coordinates a spatial topology of Minkowski spacetime is $\mathbf{S}^{2}$.

If one deals with the pure AdS geometry, then the Hilbert space representation of both theories, i.e. $\mathcal{A}_{\mathcal{M}_{1}}$ and $\mathcal{O}_{\mathcal{M}_{1}}$, are algebraically and unitarily isomorphic at the semi-classical approximation [17] (see [35] for its classical counterpart). If one further accepts an idea of

${ }^{6}$ The vector $K_{2}$ is sometimes called as a boost generator. It is misleading according to the above discussion. However, the boost generator sets indeed dynamics outside of the BTZ black hole. 


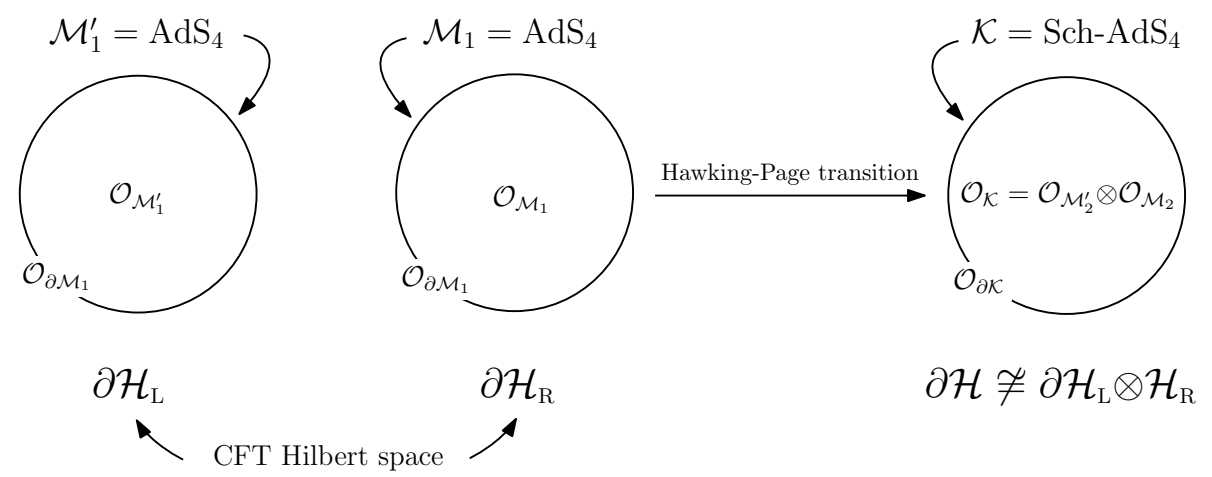

FIG. 3: A sketch of the Hawking-Page phase transition. Before the transition $\mathcal{M}_{1}$ is filled by thermal gas of particles being states in the CFT Hilbert space $\partial \mathcal{H}_{R}$. If the gas temperature $T$ exceeds the critical temperature $T_{c}$, then the nucleation of the black hole occurs. This is described at quantum level by $\mathcal{O}_{\mathcal{M}_{1}^{\prime}} \otimes \mathcal{O}_{\mathcal{M}_{1}} \rightarrow \mathcal{O}_{\mathcal{M}_{2}^{\prime}} \otimes \mathcal{O}_{\mathcal{M}_{2}}$ and $\partial \mathcal{H}_{\mathrm{L}} \otimes \partial \mathcal{H}_{R} \rightarrow \partial \mathcal{H}$. The Hilbert space $\partial \mathcal{H}$ is defined with respect to the vacuum $\left|\partial \Omega_{2}\right\rangle$ which cannot be understood in quantum field theory as an entangled state in $\partial \mathcal{H}_{\mathrm{L}} \otimes \partial \mathcal{H}_{R}$. However, it is an (maximally) entangled state in the sense it is not a product state for non-trivial field operators being products of elements from $\mathcal{O}_{\mathcal{M}_{2}^{\prime}}$ and $\mathcal{O}_{\mathcal{M}_{2}}$. In other words, the state $\left|\partial \Omega_{2}\right\rangle$ carries non-local (vacuum) correlations. This is in a complete analogy with the property of the CFT vacuum which carries non-local correlations between the "left" and "right" Rindler patch.

the eternal black-hole nucleation as pasting together two AdS spaces [36], say, $\mathcal{M}_{1}^{\prime}$ and $\mathcal{M}_{1}$, then one should associate $\partial \mathcal{H}_{\mathrm{L}}$ with the theory on $\partial \mathcal{M}^{\prime}$, and $\partial \mathcal{H}_{\mathrm{R}}$ with the theory on $\partial \mathcal{M}$. It is worth emphasising that $\partial \mathcal{H}_{\mathrm{L}, \mathrm{R}}$ are two CFT Hilbert spaces constructed on the ordinary CFT vacuum. In other words, the states $\left|\partial \Omega_{\mathrm{L}}\right\rangle$ and $\left|\partial \Omega_{\mathrm{R}}\right\rangle$ are two ordinary CFT vacua in this subsection. The thermal gas in each AdS space, i.e. $\mathcal{M}_{1}^{\prime}$ or $\mathcal{M}_{1}$, can then be obtained by considering a Kubo-Martin-Schwinger (KMS) or thermal state defined with respect to $L_{05}\left(=K_{1}=K_{2}\right)$ in each space. ${ }^{7}$ Thus, the thermal gas is composed of excitations identified with particle states in $\partial \mathcal{H}_{\mathrm{L}} \otimes \partial \mathcal{H}_{\mathrm{R}}$ (see fig. 3).

However, if the black hole has appeared through the Hawking-Page transition, then the Hilbert space of the total system should be $\partial \mathcal{H}$ which, as argued above, is not a factorized product of $\partial \mathcal{H}_{\mathrm{L}}$ and $\partial \mathcal{H}_{\mathrm{R}}$. The state $\left|\partial \Omega_{2}\right\rangle$ on $\partial \mathcal{M}$ is a pure state. It responses as a thermal state for the CFT operators having zero support on $\partial \mathcal{M}^{\prime} .8$ The same occurs for the CFT operators on $\partial \mathcal{M}^{\prime}$ which have zero support on $\partial \mathcal{M}$.

7 The KMS state $\left|\Omega_{\beta}\right\rangle$ is a state which satisfies the KMS condition: $\left\langle\Omega_{\beta}\left|\alpha_{K}^{t}(\hat{A}) \hat{B}\right| \Omega_{\beta}\right\rangle=\left\langle\Omega_{\beta}\left|\hat{B} \alpha_{K}^{t+i \beta}(\hat{A})\right| \Omega_{\beta}\right\rangle$, where both sides are analytic in the strip $0<\operatorname{Im}(t)<\beta$, continuous on its boundary, and $\alpha_{K}^{t}(\hat{A}) \equiv$ $\exp (+i \hat{K} t) \hat{A} \exp (-i \hat{K} t), \hat{K}$ is a Hermitian operator corresponding to the Killing vector $K$. More details can be found, for instance, in [18].

${ }^{8}$ This can be also phrased as follows: $\left|\partial \Omega_{2}\right\rangle$ restricted to $\partial \mathcal{M}$ (or $\partial \mathcal{M}^{\prime}$ ) is a KMS state with respect to the Killing vector $L_{05}$. 
Thus, the Hawking-Page phase transition should be understood as the evolution of the CFT operator algebra representation from $\partial \mathcal{H}_{\mathrm{L}} \otimes \partial \mathcal{H}_{\mathrm{R}}$ to $\partial \mathcal{H}$. Note that for local quantum field theory characterized by $\mathcal{A}_{\mathcal{M}}$, one thus has its representation $\mathcal{H}_{\mathrm{L}} \otimes \mathcal{H}_{\mathrm{R}}$ in $\mathcal{M}=\mathcal{M}_{1}^{\prime} \cup \mathcal{M}_{1}$ and $\mathcal{H}$ in $\mathcal{M}=\mathcal{M}_{2}^{\prime} \cup \mathcal{M}_{2}$, respectively, where $\mathcal{H}_{\mathrm{L}, \mathrm{R}}$ are here two AdS Hilbert spaces built on the ordinary AdS vacuum, and $\mathcal{H}$ is the Hilbert space defined in Sec. IIA.

To make the above statements more clear, one should restrict the consideration, for instance, to $\partial \mathcal{M}$. The physical degrees of freedom could be represented by rays either in $\partial \mathcal{H}_{\mathrm{R}}$ or $\partial \mathcal{H}$ in this boundary. The Hilbert space $\partial \mathcal{H}_{\mathrm{R}}$ is the Gelfand-Naimark-Segal representation built on $\left|\partial \Omega_{\mathrm{R}}\right\rangle$, while $\partial \mathcal{H}$ on $\left|\partial \Omega_{2}\right\rangle$. The thermal gas in $\partial \mathcal{M}$ corresponds to a "thermalized" version of $\left|\partial \Omega_{\mathrm{R}}\right\rangle$ and described by a thermal density matrix $\hat{\rho}_{\mathrm{R}}$. However, the pure state $\left|\partial \Omega_{2}\right\rangle$ responses as a thermal state merely when probed by operators $\mathcal{O}_{\partial \mathcal{K}}$ restricted to $\partial \mathcal{M}$ (or having zero support in $\partial \mathcal{M}^{\prime}$ ), i.e. $\mathcal{O}_{\partial \mathcal{M}}\left(\right.$ or $\left.\mathcal{O}_{\partial \mathcal{M}^{\prime}}\right)$. Only in the latter case one has the Tomita-Takesaki construction (e.g., see [18]) which is broadly exploited to describe physics inside of the AdS black hole by the CFT operators (see below).

Moreover, one should consider each of $\partial \mathcal{M}^{\prime}$ and $\partial \mathcal{M}$ as two Lorentzian patches of complexified Minkowski spacetime. This can be understood in the case of the spatially flat Minkowski coordinates as follows. The transition from the "right" Minkowski space to the "left" Minkowski space is achieved through $t \rightarrow-t-i \beta / 2$, where $t$ is the Minkowski time coordinate for which its spatial topology is $\mathbf{R}^{2}$. Indeed, the line element $d s^{2}=d t^{2}-d r^{2}-r^{2} d \theta^{2}$ can be written as $e^{-2 \kappa r}\left(d T^{2}-d R^{2}\right)-r^{2} d \theta^{2}$, where $T=e^{\kappa r} \sinh \kappa t$ and $R=e^{\kappa r} \cosh \kappa t>0$. One can thus analytically extend Minkowski space by allowing $R<0$. It is achieved through $t \rightarrow-t-i \beta / 2=-t-i \pi / \kappa$. More concretely, the Minkowski time coordinate has an imaginary part lying in a circle $\mathbf{S}$ with a circumference $\beta$. One may further imagine Euclidean Minkowski space, i.e. $t=i \tau$, where the Euclidean time $\tau \in \mathbf{S}$. One then considers an Euclidean wave functional of a non-interacting CFT model, i.e.

$$
\Psi[\mathcal{O}(\mathbf{y})]=\int \mathcal{D O} \exp \left(-\int_{\tau_{\mathrm{R}}}^{\tau_{\mathrm{L}}} d \tau \int d \mathbf{y}\left((\partial \mathcal{O})^{2}+(1 / 8) R \mathcal{O}^{2}\right)\right),
$$

where $y^{\mu}=(\tau, \mathbf{y}) \in \partial \mathcal{M}$, and $\tau_{\mathrm{L}}=\tau_{\mathrm{R}}+\beta / 2$. The wave functional can then be rewritten as

$$
\Psi[\mathcal{O}(\mathbf{y})]=\frac{1}{Z^{\frac{1}{2}}} \prod_{\mathbf{k}} \sum_{n=0}^{+\infty} e^{-\beta E_{\omega, n} / 2} \Psi_{\omega, n}\left[\mathcal{O}_{\mathrm{L}}(\mathbf{y})\right] \times \Psi_{\omega, n}\left[\mathcal{O}_{\mathrm{R}}(\mathbf{y})\right] .
$$

where $\omega=|\mathbf{k}|, \mathbf{k}=\left(k_{x}, k_{y}, k_{z}\right), \mathcal{O}_{\mathrm{L}, \mathrm{R}}(\mathbf{y})=\left.\mathcal{O}(y)\right|_{\tau_{\mathrm{L}, \mathrm{R}}}$ and $\Psi_{\omega, n}\left[\mathcal{O}_{\mathrm{L}, \mathrm{R}}(\mathbf{y})\right]=\left\langle\mathcal{O}_{\mathrm{L}, \mathrm{R}}(\mathbf{y}) \mid \partial n_{\mathrm{L}, \mathrm{R}}\right\rangle$. The "left" and "right" quantities refer to "left" $(R<0)$ and "right" $(R>0)$ Minkowski spaces, respectively. The connection between the path integral in non-trivial Euclidean backgrounds with the thermal-field dynamics was pointed out in [37].

Analogous formula probably exists if one introduces the so-called closed coordinates in which a spatial topology of Minkowski space is $\mathbf{S}^{2}$ (after conformally mapping it to the closed Einstein static universe). The momentum $\mathbf{k}$ is then substituted by $\omega_{\tilde{n}}=2 \tilde{n}+l+\Delta, l$ and $m$, where $\tilde{n} \in \mathbf{N}_{0}$ and $\Delta$ is a conformal weight of $\hat{\mathcal{O}}(y)$. However, the mapping between spaces is given by $t_{\mathrm{S}} \rightarrow-t_{\mathrm{S}}-i \beta / 2$ in this case. It is worth noting that $t \neq t_{\mathrm{S}}$. 
To sum it up, the algebras $\mathcal{O}_{\partial \mathcal{M}^{\prime}}$ and $\mathcal{O}_{\partial \mathcal{M}}$ should be treated as subalgebras of a larger one, i.e. $\mathcal{O}_{\partial \mathcal{K}}=\mathcal{O}_{\partial \mathcal{M}^{\prime}} \otimes \mathcal{O}_{\partial \mathcal{M}}$. In other words, these two CFT algebras should be understood as its factor subalgebras. The algebras $\mathcal{O}_{\partial \mathcal{M}^{\prime}}$ and $\mathcal{O}_{\partial \mathcal{M}}$ represented on the product Hilbert space $\partial \mathcal{H}_{\mathrm{L}} \otimes \partial \mathcal{H}_{\mathrm{R}}$ built on $\left|\partial \Omega_{\mathrm{L}}\right\rangle \otimes\left|\partial \Omega_{\mathrm{R}}\right\rangle$ can be considered as two independent copies of CFT, while the representation $\partial \mathcal{H}$ constructed on the pure state $\left|\partial \Omega_{2}\right\rangle$ can be only treated as associated with the single, enlarged CFT algebra $\mathcal{O}_{\partial \mathcal{K}}$. Thus, one should describe the bulk degrees of freedom by the single CFT algebra $\mathcal{O}_{\partial \mathcal{K}}$ represented on $\partial \mathcal{H}$. The important point is that $\partial \mathcal{H}$ is unitarily inequivalent to $\partial \mathcal{H}_{\mathrm{L}} \otimes \partial \mathcal{H}_{\mathrm{R}}$ or, in other words, $\left|\partial \Omega_{2}\right\rangle \notin \partial \mathcal{H}_{\mathrm{L}} \otimes \partial \mathcal{H}_{\mathrm{R}}$.

\section{ALGEBRAIC INEQUIVALENCE OF $\mathcal{A}_{\mathcal{M}_{2}}$ AND $\mathcal{O}_{\mathcal{M}_{2}}$}

\section{A. Inside of AdS black hole}

A construction of the bulk CFT operators is achieved in practice through the so-called smearing function $K_{\mathcal{M}}(x, y)$ which is a kernel of an integral transform, i.e.

$$
\hat{\mathcal{O}}_{\mathcal{M}}(x)=\int d^{3} y \sqrt{-g(y)} K_{\mathcal{M}}(x, y) \hat{\mathcal{O}}(y),
$$

where $x \in \mathcal{M}$ and $y \in \partial \mathcal{M}$. The smearing function was explicitly constructed in $\operatorname{AdS}$ spacetime, i.e. $\mathcal{M}=\mathcal{M}_{1}$, in [15, 16]. The algebraic and unitary equivalence of $\mathcal{A}_{\mathcal{M}_{1}}$ and $\mathcal{O}_{\mathcal{M}_{1}}$ represented on the AdS vacuum and the ordinary CFT vacuum, respectively, can already be seen at the level of two-point correlation functions of each theory. It may also be expected that $\mathcal{A}_{\mathcal{K}}$ and $\mathcal{O}_{\mathcal{K}}$ represented on the Hartle-Hawking vacuum $\left|\Omega_{2}\right\rangle$ and the state $\left|\partial \Omega_{2}\right\rangle$, respectively, describe equivalent theories.

The Tomita-Takesaki theorem is extensively exploited to describe physics inside the eternal Schwarzschild-AdS black hole. ${ }^{9}$ This theorem implies that the algebra $\mathcal{A}_{\mathcal{M}_{2}}$ acting on the Hartle-Hawking state should generate a space which is dense in $\mathcal{H}$. In other words, an observer inhabiting a region outside of the black hole should be able to probe the whole Hilbert space $\mathcal{H}$ with an arbitrary precision. This can be understood as a Reeh-Schlieder property of von Neumann algebra $\mathcal{A}_{\mathcal{M}_{2}}$. Although this property seems not to be rigorously proven in the AdS-Schwarzschild space yet, it is analogous to that in the eternal Schwarzschild geometry [21]. ${ }^{10}$ This turns out to be possible, partially because $\mathcal{A}_{\mathcal{M}_{2}}$ and $\mathcal{A}_{\mathcal{M}_{2}^{\prime}}$ considered as von Neumann algebras can be mapped into each other by an anti-unitary (modular conjugation)

\footnotetext{
${ }^{9}$ The theorem states in particular that von Neumann algebra $\mathcal{R}$ (e.g. $\mathcal{A}_{\mathcal{M}_{2}}$ ) represented in a cyclic and separating state $|\Omega\rangle$ (e.g. $\left|\Omega_{2}\right\rangle$ ) can be mapped to its commutant $\mathcal{R}^{\prime}$ (e.g. $\mathcal{A}_{\mathcal{M}_{2}^{\prime}}$ ) by an anti-unitary operator $J$. For a full formulation of the theorem, see [18]. A von Neumann algebra is an algebra of bounded operators on a Hilbert space which is closed in the weak topology of matrix elements. For example, operators of the form $\exp (\hat{\Phi}(f))$ are its elements.

${ }^{10}$ For establishing the Reeh-Schlieder property for certain subalgebras represented in the AdS vacuum in anti-de Sitter space, see [38] and [39].
} 


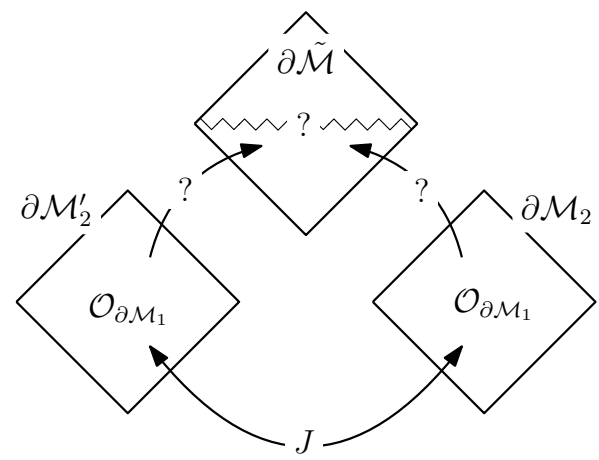

FIG. 4: A sketch of the boundary of the eternal Schwarschild-AdS black hole.

operator $J$. This operator corresponds to the transformation $t_{\mathrm{S}} \rightarrow-t_{\mathrm{S}}-i \beta / 2$ in spacetime $\mathcal{K}$ discussed above, so that

$$
J \mathcal{A}_{\mathcal{M}_{2}} J=\mathcal{A}_{\mathcal{M}_{2}^{\prime}}
$$

and vise versa. It is worth emphasising that the theorem is realised for the particular state generating the Hilbert space $\mathcal{H}$, rather than the factorised product $\mathcal{H}_{\mathrm{L}} \otimes \mathcal{H}_{\mathrm{R}}$.

The same construction should be employed on the AdS boundary if one expects the (extrapolate) AdS/CFT correspondence in AdS space with a Schwarzschild black hole. The Tomita-Takesaki theorem is applicable if one works with the Hilbert space representation $\partial \mathcal{H}$ of $\mathcal{O}_{\partial \mathcal{K}}$ constructed with respect to the state $\left|\partial \Omega_{2}\right\rangle$. To construct CFT operators inside of the black hole, one needs to extend the boundary by introducing extra Minkowski space $\partial \tilde{\mathcal{M}}$. This Minkowski space in flat coordinates corresponds to $T>0$ and $T>R$. It is worth noting that $\partial \mathcal{M}^{\prime}, \partial \mathcal{M}$ and $\partial \tilde{\mathcal{M}}$ are not separated by any horizons. However, it is not evident whether this extra geometrical construction is acceptable. This question would be interesting to investigate in detail.

It is worth noting that $\partial \tilde{\mathcal{M}}$ is a half of the Milne patch of Minkowski space in the case of the BTZ black hole (up to the quotient). This patch is a part of the whole boundary of $\mathrm{AdS}_{3}$. Thus, no extra geometrical construction is needed in the BTZ black-hole case.

To summarise, it is necessary to understand the boundary structure of the SchwarzschildAdS black hole to implement the idea of AdS/CFT in this geometry. This could be a way towards finding a proper mapping of the CFT operators inside the black hole.

\section{B. No-Go theorem}

The correspondence principle implies that there is at least algebraic isomorphism between $\mathcal{A}_{\mathcal{K}}$ and $\mathcal{O}_{\mathcal{K}}$ in the large $N$ limit. To have a unitary isomorphism between them, their Hilbert space representations must by isomorphic (equal up to a unitary transform). Both representations are obtained through the Gelfand-Naimark-Segal construction [18] built on 
the pure states $\left|\Omega_{2}\right\rangle$ and $\left|\partial \Omega_{2}\right\rangle$. These states are KMS (thermal) ones with respect to the same Killing vector $L_{05} \in \mathfrak{s o}(2,3)$ and, e.g., subalgebras $\mathcal{A}_{\mathcal{M}_{2}}$ and $\mathcal{O}_{\mathcal{M}_{2}}$, respectively.

It is worth emphasising at this point that the KMS condition depends on two ingredients. These are a field operator algebra and a one-parameter group of its automorphism. An empty state can also satisfy the KMS condition depending on the operator algebra and its automorphism chosen to probe this state. For example, the Minkowski vacuum is a KMS state with respect to an operator subalgebra with vanishing support in one of the Rindler patches and its automorphism generated by the boost Killing vector [20, 21]. In the case of a non-interacting, massless scalar field model, the Minkowski vacuum is also a (conformal) KMS state with respect to an operator subalgebra with vanishing support either in the contracting or in the expanding Milne patch and its automorphism generated by the dilatation (see, for instance, [40]).

Non-uniqueness of a KMS state can appear through the existence of several distinct thermodynamic phases [18]. This can be exemplified by the Hawking-Page phenomenon [25]. Indeed, the same inverse temperature $\beta$ characterises two different states, namely the AdS space with the thermal gas and the Hartle-Hawking state restricted to the region outside of the black hole. In the path integral approach in the saddle-point approximation, both phases are coexisting, described by a non-extremal KMS state ${ }^{11}$ and can be distinguished by an appropriately designed observable [41].

Non-uniqueness of a KMS state can also appear through a spontaneous symmetry breaking [18]. For example, one may construct two KMS states with the same inverse temperature under two states one of which is symmetric under $\mathrm{SU}(2)$ and another under $\mathrm{U}(1)$ (a phase of the spontaneous magnetisation).

Although both states, the AdS vacuum at the Hawking-Page temperature and the HartleHawking vacuum, are invariant under the same subalgebra of $\mathfrak{s o}(2,3)$, namely $\mathbf{R} \oplus \mathfrak{s o}(3)$, these vacua are different. This originates from different topologies of $\mathcal{M}_{1}$ and $\mathcal{M}_{2}$. The topology of the boundary is however unchanged during the Hawking-Page transition. Moreover, it is the same Minkowski space. Therefore, the KMS state defined with respect to $L_{05}$ on the boundaries of both $\mathcal{M}_{1}$ and $\mathcal{M}_{2}$ is unique, i.e. the thermal two-point function does not change for the boundary CFT theory.

A few remarks are in order. First, the effective field theory we are considering in the bulk, i.e. $\mathcal{A}_{\mathcal{M}}$, is non-interacting. According to the holographic idea, there should exist a certain free or Gaussian CFT theory on the boundary which may or may not correspond to $\mathcal{A}_{\mathcal{M}}$. In the case of AdS space, we specified this CFT and denoted it as $\mathcal{O}_{\partial \mathcal{M}}$. Second, the CFT algebra $\mathcal{O}_{\partial \mathcal{M}}$ is also known as being CFT at infinite $N$ [42]. One can treat a generalised free field operator $\hat{\mathcal{O}}_{\partial \mathcal{M}} \in \mathcal{O}_{\partial \mathcal{M}}$ as composed of some complicated operators of a strongly coupled CFT which is dual to a full UV complete theory in AdS (e.g., see [43]). Thus,

11 An extremal KMS state is a state that cannot be decomposed into a mixture of other KMS states. 
the uniqueness of the KMS state is related to the operator algebra $\mathcal{O}_{\partial \mathcal{M}}$, i.e. the effective non-interacting CFT.

The thermal two-point function can be obtained from the causal propagator [44, 45]:

$$
\left\langle\Omega_{\beta}\left|\hat{\Phi}_{\mathcal{M}}(x) \hat{\Phi}_{\mathcal{M}}\left(x^{\prime}\right)\right| \Omega_{\beta}\right\rangle=\frac{i}{2 \pi} \int_{\mathbf{R}} \frac{d k}{1-e^{-\beta k}} \int_{\mathbf{R}} d \delta x^{0} e^{i k \delta x^{0}} \Delta_{\mathcal{M}}\left(x^{0}+\delta x^{0}, \mathbf{x} \mid x^{\prime}\right),
$$

where $\beta$ is the inverse temperature, and $\left|\Omega_{\beta}\right\rangle$ is a KMS state defined with respect to a Killing vector generating a translation along $x^{0}$. The causal propagator, i.e. $\Delta_{\mathcal{M}_{1}}\left(x, x^{\prime}\right)$, is given by a difference between the retarded and advanced Green function of a field equation satisfied by $\Phi_{\mathcal{M}}(x)$. This propagator depends on a background geometry (stressed out by the lower index $\mathcal{M}$ ) as well as boundary conditions. In quantum field theory it provides the commutator of the field operator at two spacetime points, i.e.

$$
i \Delta_{\mathcal{M}_{1}}\left(x, x^{\prime}\right)=\left[\hat{\Phi}_{\mathcal{M}_{1}}(x), \hat{\Phi}_{\mathcal{M}_{1}}\left(x^{\prime}\right)\right]
$$

(assuming $\hat{\Phi}(x)$ is non-interacting, i.e. $N$ is infinite). It is worth noting that $\Delta_{\mathcal{M}_{1}}\left(x, x^{\prime}\right)$ is state-independent. After a certain rescaling of the field operator, $\Delta_{\mathcal{M}_{1}}\left(x, x^{\prime}\right)$ reduces to a CFT causal propagator in AdS space on its boundary, namely

$$
\Delta_{\partial \mathcal{M}}\left(y, y^{\prime}\right)=\lim _{r \rightarrow \infty} r^{2 \Delta} \Delta_{\mathcal{M}_{1}}\left(y, r ; y^{\prime}, r\right)
$$

where $\Delta$ is a conformal weight of $\hat{\mathcal{O}}(y)(y \in \partial \mathrm{AdS})$ corresponding to $\hat{\Phi}_{\mathcal{M}_{1}}(x)$ in the bulk of AdS, and $\Delta_{\partial \mathcal{M}}\left(y, y^{\prime}\right)=-i\left[\hat{\mathcal{O}}(y), \hat{\mathcal{O}}\left(y^{\prime}\right)\right]$. The thermal two-point function of the boundary CFT operators depends on a Killing vector chosen to construct this state. The KMS state defined with respect to $L_{05}$ (the AdS global time translation generator) on the boundary is thus identical to the thermal gas of AdS particles in the bulk. In the limit of vanishing temperature, the KMS state thus defined will approach the ordinary AdS vacuum in the bulk and the ordinary CFT vacuum on the boundary.

During the Hawking-Page transition, the causal propagator of the field $\hat{\Phi}_{\mathcal{M}}(x)$ changes as the global structure of space changes. In other words, $\Delta_{\mathcal{M}_{1}}\left(x, x^{\prime}\right) \neq \Delta_{\mathcal{M}_{2}}\left(x, x^{\prime}\right)$ as it can be seen, for example, if one compares anti-symmetric combination of two-point correlation functions in $\mathcal{M}_{1}$ and $\mathcal{M}_{2}$ for a scalar field model conformally coupled to gravity [41]. However, the boundary is still compactified Minkowski space and the CFT algebra is still invariant under the conformal algebra $\mathfrak{s o}(2,3)$. In other words, $\Delta_{\partial \mathcal{M}}\left(y, y^{\prime}\right)$ cannot be obtained from (14) with $\Delta_{\mathcal{M}_{2}}\left(x, x^{\prime}\right)$ instead of $\Delta_{\mathcal{M}_{1}}\left(x, x^{\prime}\right)$ on the right-hand side. Indeed, one has

$$
\lim _{r \rightarrow \infty} r^{2 \Delta} \Delta_{\mathcal{M}_{1}}\left(y, r ; y^{\prime}, r\right) \propto \frac{1}{\cos \Delta \Theta-\cos \left(\Delta t_{\mathrm{S}}-i \varepsilon\right)}-\frac{1}{\cos \Delta \Theta-\cos \left(\Delta t_{\mathrm{S}}+i \varepsilon\right)}
$$

where $y=\left(t_{\mathrm{S}}, \theta, \phi\right), \cos \Delta \Theta \equiv \cos \theta \cos \theta^{\prime}+\sin \theta \sin \theta^{\prime} \cos \left(\phi-\phi^{\prime}\right)$ and $H_{\mathrm{AdS}} \equiv 1$, whereas

$$
\lim _{r \rightarrow \infty} r^{2 \Delta} \Delta_{\mathcal{M}_{2}}\left(y, r ; y^{\prime}, r\right) \propto \frac{2 \Delta \Theta}{\sin \Delta \Theta}\left(\frac{1}{\Delta \Theta^{2}-\left(\Delta t_{\mathrm{S}}-i \varepsilon\right)^{2}}-\frac{1}{\Delta \Theta^{2}-\left(\Delta t_{\mathrm{S}}+i \varepsilon\right)^{2}}\right)
$$

in the case, e.g., of the Neumann boundary condition (see [41] for more details). 
It is usually assumed that it is enough to have a pure high-energy state on the boundary theory to reproduce local quantum field theory in the bulk of the Schwarzschild-AdS black hole. As it should be evident from the above discussion, one has at least to supplement that condition by a requirement (14) is also satisfied for $\mathcal{M}_{2}$. Since this supplementary condition is not realised in the present case, there is no algebraic isomorphism between the operators $\mathcal{A}_{\mathcal{K}}$ and $\mathcal{O}_{\mathcal{K}}$.

One is hence forced to conclude by a sort of no-go theorem:

A description of physics outside/inside of the eternal Schwarzschild-AdS black hole is inconsistent with either the correspondence principle or the holographic principle.

A few remarks are in order. The holographic principle is here understood in a narrow sense, namely in terms of the (extrapolate) AdS/CFT idea. Accordingly, the fundamental part of the set-up is the conformal, strongly coupled QFT on the boundary. Its subalgebra, i.e. $\mathcal{O}_{\mathcal{M}}$, which can be treated as being non-interacting at $N \rightarrow \infty$, should reproduce results based on an effective local QFT, i.e. $\mathcal{A}_{\mathcal{M}}$, defined on a semi-classical background $\mathcal{M}$ as expected from the correspondence principle. The consistency of these principles is well-established in AdS, whereas the modification of the CFT algebra $\mathcal{O}_{\mathcal{M}_{2}}$ is required in Schwarzschild-AdS space as $\mathcal{A}_{\mathcal{M}_{2}}$ does not acquire conformal symmetry at the operator level on the boundary. One can insist on having $\mathcal{O}_{\mathcal{M}_{2}}$, but then this theory must be singular near the black-hole horizon.

\section{CONCLUDING REMARKS}

CFT Hilbert space representations. The first point we would like to emphasiae is that one cannot describe the AdS black hole by two independent CFTs if one expects the (extrapolate) AdS/CFT correspondence in the AdS black-hole geometry. This follows from the Hilbert space representation that has to be chosen for the CFT algebras on the "left" and "right" eternal AdS black-hole boundaries. This representation is $\partial \mathcal{H}$, not the factorised product $\partial \mathcal{H}_{\mathrm{L}} \otimes \partial \mathcal{H}_{\mathrm{R}}$. These CFT algebras, $\mathcal{O}_{\partial \mathcal{M}^{\prime}}$ and $\mathcal{O}_{\partial \mathcal{M}}$, should then be treated as factor subalgebras of the single $\mathrm{CFT}$ algebra $\mathcal{O}_{\partial K}$. It is worth stressing that this construction is state-dependent. Note that it is in agreement only to a certain extent with [43].

For example, the two CFT algebras on each boundary of the BTZ black hole are factors of a single CFT algebra represented on the ordinary CFT vacuum (up to the quotient). The Gelfand-Naimark-Segal representation of the CFT algebra on this state is not unitarily equivalent to the factorised product of two CFT Hilbert spaces defined on each BTZ boundary. This is in disagreement with [8, 27], but resolves a problem posed in [34] of no holographic description of the BTZ black hole.

An understanding of the Hawking-Page phase transition as the change of the Hilbert 
space representation of the $\mathrm{CFT}$ algebra $\mathcal{O}_{\partial \mathcal{M}^{\prime}} \otimes \mathcal{O}_{\partial \mathcal{M}}$ seems to be reasonable in the light of [46]. Indeed, the QCD phase transition is characterised by a change of the representation. This has its imprint in the change of the notion of quasi-particles at the QCD energy scale $\Lambda_{\mathrm{QCD}} \approx 0.3 \mathrm{GeV}$. At low energies, i.e. below $\Lambda_{\mathrm{QCD}}$, these particles are protons, neutrons, mesons and other hadrons, but at high-energy regime, i.e. at energies well above $\Lambda_{\mathrm{QCD}}$, these are gluons and quarks.

In Sec. IIIB we have argued that the KMS state defined w.r.t. $L_{05}$ for the free CFT is unique. The phase transition $\partial \mathcal{H}_{\mathrm{L}} \otimes \partial \mathcal{H}_{\mathrm{R}} \rightarrow \partial \mathcal{H}$ does not change the correlation function of the CFT operator $\hat{\mathcal{O}}(y)$ at two separate boundary points. This can be understood as follows. Before the phase transition the CFT in the thermal bath of excitations defined w.r.t. to the CFT vacuum $\left|\partial \Omega_{1}\right\rangle$ and characterised by a density matrix $\hat{\rho}$. After the phase transition the $\mathrm{CFT}$ is in the pure state $\left|\partial \Omega_{2}\right\rangle$. However, one has

$$
\operatorname{Tr}\left(\hat{\rho} \hat{\mathcal{O}}(y) \hat{\mathcal{O}}\left(y^{\prime}\right)\right)=\left\langle\partial \Omega_{2}\left|\hat{\mathcal{O}}(y) \hat{\mathcal{O}}\left(y^{\prime}\right)\right| \partial \Omega_{2}\right\rangle
$$

where the trace on the right-hand side is taken w.r.t. states of the CFT Hilbert space $\partial \mathcal{H}_{R}$. Note that $\left|\partial \Omega_{2}\right\rangle$ provides a vacuum representation of $\mathcal{O}_{\partial \mathcal{K}}$ and is a KMS state when probed by $\mathcal{O}_{\partial \mathcal{M}}$.

If one rejects the representation evolution, then one could not holographically describe an interior of the black hole. This results from the fact that the Tomita-Takesaki theorem broadly exploited to construct CFT operators inside the hole is realised only if the CFT algebra is represented in $\partial \mathcal{H}$, not in $\partial \mathcal{H}_{\mathrm{L}} \otimes \partial \mathcal{H}_{\mathrm{R}}$. However, this also requires an extra geometric construction on the boundary (see Sec. IIIA).

Algebraic inequivalence of $\mathcal{A}_{\mathcal{K}}$ and $\mathcal{O}_{\mathcal{K}}$. One may expect that it is enough to choose a "proper" CFT state defined on the CFT algebra to have a smooth horizon in the bulk (see, for instance, [43]). However, this expectation is based on an implicit assumption that $\mathcal{A}_{\mathcal{K}}$ and $\mathcal{O}_{\mathcal{K}}$ are algebraically isomorphic in the large $N$ limit. As shown in IIIB, this assumption is invalid.

It is worth noting that this algebraic isomorphism exists in the case of the BTZ black hole. The essential reason for this is that the BTZ black hole locally corresponds to anti-de Sitter space, i.e. possesses the full Killing algebra of AdS space.

Presumably, the no-go theorem formulated in Sec. IIIB can be generalised to the Schwarzschild-AdS black hole formed through the gravitational collapse. It should be possible, because the main argument is based on comparison the algebraic structure of the semi-classical quantum field operators with that of the CFT ones which depend only on the spacetime metric.

It is worth further noting that the high-energy pure state $|E\rangle$ employed in [43] is of different nature in comparison with $\left|\partial \Omega_{2}\right\rangle$. The choice of this high-energy state $|E\rangle$ leads merely to the spontaneous AdS symmetry breaking in the large $N$ limit (in the sense of [47]). Modelling a change of spacetime isometry during $\mathcal{M}_{1} \rightarrow \mathcal{M}_{2}$ by the spontaneous symmetry 
breaking on the boundary seems to be impossible, because the algebraic structure of the field operators depends on a geometry, but not on a state.

Indeed, the state $|E\rangle$ is obtained from the CFT vacuum $\left|\partial \Omega_{1}\right\rangle$ by acting on it by a certain CFT operator $\hat{O} \in \mathcal{O}_{\partial \mathcal{M}}:|E\rangle=\hat{O}\left|\partial \Omega_{1}\right\rangle$. The operator $\hat{O}$ is unitary and well-defined or nonanomalous when applied to $\left|\partial \Omega_{1}\right\rangle$. Thus, the Heisenberg and Schrödinger pictures should be equivalent. Therefore, instead of introducing a new state $|E\rangle$ on $\mathcal{O}_{\partial \mathcal{M}}$, one can consider a new CFT algebra $\mathcal{O}_{\partial \mathcal{M}}^{\prime} \equiv \hat{O} \mathcal{O}_{\partial \mathcal{M}} \hat{O}^{\dagger}$. The new algebra $\mathcal{O}_{\partial \mathcal{M}}^{\prime}$ is isomorphic to $\mathcal{O}_{\partial \mathcal{M}}$ even at finite $N$. If one does not take this into account, then one has a pathological theory (e.g., no bulk covariance) in the bulk even in AdS space [48].

One may consider the CFT bulk operators in the AdS black-hole backgrounds as the only available observables. This would be in the spirit of the holography [1, 2]. However, the bulk CFT operators must be singular on the black-hole horizon, because $\mathcal{A}_{\mathcal{K}} \neq \mathcal{O}_{\mathcal{K}}$ even in the semi-classical limit. This has been actually observed in [49].

\section{ACKNOWLEDGMENTS}

It is a pleasure to thank D. Buchholz, C. Germani, S. Konopka, D. Ponomarev, A. Vikman for discussions. I am also thankful to R. Verch for discussions and the reference [21]. I am indebted to M. Haack for discussions and his valuable comments on an early version of this paper. We are grateful to the anonymous referee for his comments/questions/suggestions which helped to substantially improve the presentation of the paper. This research is supported by TRR 33 "The Dark Universe".

[1] G. t' Hooft, "Dimensional reduction in quantum gravity," arXiv:gr-qc/9310026.

[2] L. Susskind, "The world as a hologram," J. Math. Phys. 36, 6377 (1995) arXiv:hep-th/9409089.

[3] J. Maldacena, "The large $N$ limit of superconformal field theories and supergravity," Adv. Theor. Math. Phys. 2, 231 (1998); arXiv:hep-th/9711200.

[4] S.S. Gubser, I.R. Klebanov, A.M. Polyakov, "Gauge theory correlators from non-critical string theory," Phys. Lett. B428, 105 (1998); arXiv:hep-th/9802109.

[5] E. Witten, "Anti-de Sitter space and holography," Adv. Theor. Math. Phys. 2, 253 (1998); arXiv:hep-th/9802150.

[6] V. Balasubramanian, P. Kraus, A. Lawrence, "Bulk versus boundary dynamics in anti-de Sitter spacetime," Phys. Rev. D59, 046003 (1999); arXiv:hep-th/9805171.

[7] T. Banks, M.R. Douglas, G.T. Horowitz, E. Martinec, "AdS dynamics from conformal field thoery," arXiv:hep-th/9808016. 
[8] V. Balasubramanian, P. Kraus, A. Lawrence, S.P. Trivedi, "Holographic probes of anti-de Sitter spacetimes," Phys. Rev. D59, 104021 (1999); arXiv:hep-th/9808017.

[9] S.B. Giddings, "(Non)perturbative gravity, nonlocality, and nice slices," Phys. Rev. D74, 106009 (2006); arXiv:hep-th/0606146.

[10] S.B. Giddings, "The gravitational S-matrix: Erice lectures," Subnucl. Ser. 48, 93 (2013); arXiv:hep-th/1105.2036.

[11] S.B. Giddings, "Hilbert space structure in quantum gravity: an algebraic perspective," JHEP12, 099 (2015); arXiv:hep-th/1503.08207.

[12] S. Hollands, R.M. Wald, "Quantum field theory in curved spacetime," arXiv:gr-qc/1401.2026.

[13] I. Khavkine, V. Moretti, "Algebraic QFT in curved spacetime and quasi-free Hadamard states: an introduction", arXiv:math-ph/1412.5945.

[14] Ch.J. Fewster, R. Verch, "Algebraic quantum field theory in curved spacetimes," arXiv:math$\mathrm{ph} / 1504.00586$.

[15] I. Bena, "Construction of local fields in the bulk of $\mathrm{AdS}_{5}$ and other spaces," Phys. Rev. D62, 066007 (2000), arXiv:hep-th/9905186.

[16] A. Hamilton, D. Kabat, G. Lifschytz, D.A. Lowe, "Local bulk operators in AdS/CFT correspondence: a boundary view of horizons and locality," Phys. Rev. D73, 086003 (2006), arXiv:hep-th/0506118; "Holographic representation of local bulk operators," Phys. Rev. D74, 066009 (2006), arXiv:hep-th/0606141; "Local bulk operators in AdS/CFT correspondence: a holographic description of the black hole interior," Phys. Rev. D75, 106001 (2007), arXiv:hep-th/0612053.

[17] K.H. Rehren, "Local quantum observables in the anti-de Sitter/conformal QFT correspondence," Phys. Lett. B493, 383 (2000), arXiv:hep-th/0003120; "Algebraic holography," Ann. Henri Poincaré 1, 607 (2000), arXiv:hep-th/9905179.

[18] R. Haag, Local quantum physics. Fields, Particles, Algebras, (Springer-Verlag, 1996).

[19] W. Israel, "Thermo-field dynamics of black holes," Phys. Lett. A57, 107 (1976).

[20] G.L. Sewell, "Quantum fields on manifolds: PCT and gravitationally induced thermal states," Ann. Phys. 141, 201 (1982).

[21] B.S. Kay, "A uniqueness result for quasi-free KMS states," Helvetica Physica Acta 58, 1017 (1985); "Purification of KMS states," Helvetica Physica Acta 58, 1030 (1985); "The doublewedge algebra for quantum fields on Schwarzschild and Minkowski spacetimes," Commun. Math. Phys. 100, 57 (1985).

[22] R.M. Wald, Quantum field theory in curved spacetime and black hole thermodynamics, (Chicago University Press, 1994).

[23] J. Yngvason, "The role of type III factors in quantum field theory," Rep. Math. Phys. 55, 135 (2005); arXiv:math-ph/0411058.

[24] H. Umezawa, Advanced field theory. Micro, macro and thermal physics, (AIP Press, 1993).

[25] S.W. Hawking, D.N. Page, "Thermodynamics of black holes in anti-de Sitter space," Commun. 
Math. Phys. 87, 577 (1983).

[26] S. Emelyanov, "Can gravitational collapse and black-hole evaporation be unitary process after all?," arXiv:hep-th/1507.03025.

[27] J. Maldacena, "Eternal black holes in anti-de Sitter," JHEP04, 021 (2003), arXiv:hep-th/0106112.

[28] G. Horowitz, J. Polchinski, "Gauge/gravity duality", (in Approaches to quantum gravity. Toward a new understanding of space, time and matter, edited by D. Oriti, Cambridge University Press, 2009).

[29] S.R. Roy, D. Sarkar, "Hologram of a pure state black hole," arXiv:hep-th/1505.03895.

[30] (a) M. Bañados, C. Teitelboim, J. Zanelli, "Black hole in three-dimensional spacetime," Phys. Rev. Lett. 69, 1849 (1992); arXiv:hep-th/9204099; (b) M. Bañados, M. Henneaux, C. Teitelboim, J. Zanelli, "Geometry of the 2+1 black hole," Phys. Rev. D48, 1506 (1993); arXiv:hep-th/9302012.

[31] S. Carlip, "The (2+1)-dimensional black hole," Class. Quant. Grav. 12, 2853 (1995); arXiv:gr-qc/9506079

[32] G.T. Horowitz, D. Marolf, "A new approach to string cosmology," JHEP07, 014 (1998); arXiv:hep-th/9805207.

[33] S. Emelyanov, "Freely moving observer in (quasi) anti-de Sitter space," Phys. Rev. D90, 044039 (2014), arXiv:gr-qc/1309.3905; "Local thermal observables in spatially open FRW spaces," Phys. Rev. D91, 124068 (2015), arXiv:gr-qc/1406.3360.

[34] S.G. Avery, B.D. Chowdhury, "No holography for eternal AdS black holes," arXiv:hepth/1312.3346.

[35] B.S. Kay, P. Larkin, "Pre-holography," Phys. Rev. D77, 121501 (2008); arXiv:hepth/0708.1283.

[36] M.Van Raamsdonk, "Building up spacetime with quantum entanglement," Gen. Rel. Grav. 42, 2323 (2010), arXiv:hep-th/1005.3035.

[37] R. Laflamme, "Geometry and thermodynamics," Nucl. Phys. B324, 233 (1988).

[38] D. Buchholz, S.J. Summers, "Stable quantum systems in anti-de Sitter space: causality, independence, and spectral properties," J. Math. Phys. 45, 4810 (2004), arXiv:math-ph/0407011.

[39] I.A. Morrison, "Boundary-to-bulk maps for AdS causal wedges and the Reeh-Schlieder property in holography," JHEP05, 053 (2014), arXiv:hep-th/1403.3426.

[40] S. Emelyanov, "Non-unitarity or hidden observables?," arXiv:gr-qc/1410.6149.

[41] S. Emelyanov, "Observing quantum gravity in asymptotically AdS space," Phys. Rev. D92, 124062 (2015); arXiv:hep-th/1504.05164.

[42] J. Kaplan, "Lectures on AdS/CFT from the bottom up" (2015).

[43] K. Papadodimas, S. Raju, "An infalling observer in AdS/CFT," JHEP10, 212 (2013), arXiv:hep-th/1211.6767; "Comments on the Necessity and Implications of State-Dependence in the Black Hole Interior," arXiv:hep-th/1503.08825. 
[44] R. Haag, H. Narnhofer, U. Stein, "On quantum field theory in gravitational background," Commun. Math. Phys. 94, 219 (1984).

[45] H. Narnhofer, I. Peter, W. Thirring, "How hot is the de Sitter space?," Int. J. Mod. Phys. B10, 1507 (1996).

[46] E. Witten, "Anti-de Sitter space, thermal phase transition, and confinement in gauge theories," Adv. Theor. Math. Phys. 2, 505 (1998), arXiv:hep-th/9803131.

[47] I. Ojima, "Lorentz invariance versus temperature in QFT," Lett. Math. Phys. 11, 73 (1986).

[48] S. Emelyanov, "A note on bulk locality and covariance in AdS/CFT ," arXiv:hepth/1507.07897.

[49] D. Kabat, G. Lifschytz, "Finite $N$ and the failure of bulk locality: black holes in AdS/CFT," JHEP09, 077 (2014), arXiv:hep-th/1405.6394. 